\title{
Adiponectin Receptor-1 C-Terminal Fragment (CTF) in Plasma: Putative Biomarker for Diabetes
}

\author{
Michael J. Pugia • Deanna D. H. Franke • \\ Sean L. Barnes • Amy Zercher • David Brock • \\ Mary Foltz • Roland Valdes Jr. • Saeed A. Jortani
}

Published online: 9 September 2009

(C) Humana Press 2009

\begin{abstract}
Introduction Polypeptide fragments from cell surface receptors when found in plasma may be indicators of receptor regulation in disease conditions. It is known that subjects with diabetes have significantly lower plasma concentrations of adiponectin, a hormone released by adipose tissue, compared with nondiabetic controls. This hormone interacts with cell surface receptors in muscle (AdipoR1) and liver (AdipoR2). Methods We analyzed the relative distribution of specific fragments of AdipoR1 in healthy and diabetic individuals using an immunoaffinity mass spectrometry approach. We used antibodies raised against AdipoR1 immobilized on pre-activated protein chip surfaces to determine the molecular weights of bound polypeptide fragments using immunomass spectrometry (immuno-MS).

Results Initially, immuno-MS analyses using a polyclonal antibody revealed two peaks $(\mathrm{m} / \mathrm{z} 3,902$ and 7,812) in plasma from normal, healthy individuals $(n=5)$ that were not present in the plasma of diabetics $(n=5)$. To confirm the detection of these fragments, a monoclonal antibody was developed against the last 25 amino acids of the AdipoR1
\end{abstract}

\footnotetext{
M. J. Pugia $\cdot$ A. Zercher $\cdot$ D. Brock $\cdot$ M. Foltz

Urinalysis Research and Development,

Siemens Healthcare Diagnostics,

Elkhart, IN 46516, USA

D. D. H. Franke $\cdot$ S. L. Barnes $\cdot$ R. Valdes Jr. Department of Pathology and Laboratory Medicine, School of Medicine, University of Louisville, 511 South Floyd Street (Room 217),

Louisville, KY 40292, USA

\section{S. A. Jortani $(\triangle)$}

Department of Pathology and Laboratory Medicine,

School of Medicine, University of Louisville,

Louisville, KY 40202, USA

e-mail: sjortani@louisville.edu
}

C-terminal fragment (CTF). Using the immuno-MS method, the monoclonal antibody detected the AdipoR1 CTF $(\mathrm{m} / \mathrm{z} 3475)$ in all healthy controls $(n=10)$, but did not detect these fragments in the diabetic patients $(n=10)$.

Discussion These preliminary observations suggest that the plasma levels of this receptor fragment may serve as an indicator of diabetic condition.

Keywords Adiponectin - Adiponectin receptors . Type 2 diabetes · Insulin resistance · Proteomics · Interaction mapping

\section{Introduction}

Studies have shown decreased concentrations of adiponectin in plasma from obese individuals, and these findings have also been inversely associated with insulin resistance and a higher incidence of type 2 diabetes [1-10]. Adiponectin is a $30-\mathrm{kD}$ peptide hormone produced by adipose tissue $[11,12]$. This hormone has been shown to increase insulin sensitivity by several mechanisms [13], including inhibition of hepatic gluconeogenesis, activation of muscle to increase glucose uptake and metabolism, and activation of muscle and liver to increase fatty acid oxidation [14-16].

Adiponectin activates glucose metabolism through two principal receptors: AdipoR1, predominately expressed in skeletal muscle, and AdipoR2, predominately expressed in the liver [12]. AdipoR1 and R2 consist of a seven-transmembrane domain that is coupled to a $\mathrm{G}$ protein signaling pathway, but unlike typical $G$ protein receptors, the carboxyl terminus of these adiponectin receptors is located on the extracellular side of the membrane instead of on the intracellular side [12].

Reports indicate that AdipoR1 receptor expression, as well as adiponectin hormone concentration, is reduced in 
diabetic conditions $[17,18]$. Pugia postulated that the turnover of the receptor could be measured by quantitating the level of C-terminal fragments (CTFs) released into the blood and went on to show by enzyme-linked immunosorbent assay (ELISA) that levels of these fragments were significantly altered in diabetes and cardiovascular disease patients [19]. However, due to the limitations associated with the use of ELISA, the specific CTFs could not be distinguished.

Considering the reduction in adiponectin and its receptor in diabetes, the objective of the current study was to establish whether the degraded fragments of the receptor released in circulation of diabetics would be different from nondiabetics. We therefore analyzed the relative distribution of AdipoR1 receptor fragments in the plasma of healthy and diabetic individuals. We report that a specific AdipoR1 $\mathrm{CTF}$ is present in the plasma of normal subjects but is missing from the plasma of diabetic subjects.

\section{Methods}

\section{Patient Samples}

Heparinized plasma specimens from diabetic and normal subjects were obtained from Siemens Diagnostics (Elkhart, IN, USA). Sodium heparin plasma samples for normal patients without diabetes, high cholesterol, hypertension or metabolic syndrome, and body mass index (BMI) of $<30$ were collected in hospital and primary care settings (Continental Services Group, Miami, FL, USA). Sodium heparin plasma samples were collected for types I and II diabetics enrolled in an in-house glucose meter study who were routinely monitored by venous glucose and $\% \mathrm{HbA} 1 \mathrm{c}$. The institutional review board practices of our institutions were followed, and analysts were blinded to all tests. Specimens were aliquoted and maintained at $-70^{\circ} \mathrm{C}$ until analysis. Samples from five healthy and five diabetic patients were used for the initial discovery work using polyclonal antibody. Age, gender, and BMI demographics matched between healthy and diabetic samples selected. For the confirmation work using monoclonal antibody, additional samples from five healthy and five diabetic patients were added to the respective existing groups to yield $n=10$ for each group.

\section{Reagents: Peptide and Antibodies}

The human AdipoR1 C-terminal peptide sequence (AdipoR1 357-375: N-L-Q-E-F-R-Y-G-L-E-G-G-C-T-D-D-T-L-L) was synthesized with $\geq 90 \%$ purity by Phoenix Pharmaceuticals, Inc. (Belmont, CA, USA). The lyophilized peptide $(100 \mu \mathrm{g})$ was resuspended with $60 \%$ acetonitrile in $\mathrm{diH} 20$ with $0.1 \%$ trifluoroacetic acid and phosphate buffered saline (PBS;
$\mathrm{pH}$ 7.4). Final concentration of AdipoR 1 peptide was targeted to be $1 \mu \mathrm{g} / \mu \mathrm{L}(0.51 \mathrm{nmol} / \mu \mathrm{L})$. Stock peptide was aliquoted and maintained at $-70^{\circ} \mathrm{C}$. Peptide integrity check using a NP20 chip type followed by mass spectrometric analysis revealed mass of 2,144 $\mathrm{Da}$ that was comparable to the calculated value of 2,126 $\mathrm{Da}$. As mentioned by the manufacturer, a 4,300-Da mass was also observed, representing a peptide dimer.

Anti-Human AdipoR1 (357-375)-NH2 polyclonal IgG antibody was obtained from Phoenix Pharmaceuticals, Inc. (Belmont, CA, USA). According to the manufacturer's instructions, $200 \mu \mathrm{g}$ lyophilized antibody was resuspended with PBS ( $\mathrm{pH}$ 7.4) and $\mathrm{diH}_{2} \mathrm{O}$ to yield a final antibody concentration of $1 \mu \mathrm{g} / \mu \mathrm{L}$. Stock antibody was aliquoted and maintained at $-70^{\circ} \mathrm{C}$. Antibody integrity check using a NP20 chip type followed by mass spectrometric analysis revealed a pure antibody preparation, given a mass $\cong 143,800 \mathrm{Da}$. An anti-Human AdipoR1 monoclonal IgG antibody was obtained from Siemens Diagnostics (Elkhart, IN, USA). Clone 444-1D12.1H7 was raised against the C-terminal Adipo-R1 25 mer (H-F-Y-G-V-S-N-L-Q-E-F-R-Y-G-L-E-GG-C-T-D-D-T-L-L). Using $\mathrm{diH}_{2} \mathrm{O}$, stock antibody was diluted to a final concentration of monoclonal Adipo-R $1=1 \mu \mathrm{g} / \mu \mathrm{L}$. Stock antibody was aliquoted and maintained at $-70^{\circ} \mathrm{C}$. Antibody integrity check using a NP20 chip type followed by mass spectrometric analysis revealed a pure antibody preparation, given a mass $\cong 147,600 \mathrm{Da}$. Anti-Human Troponin I monoclonal IgG antibody (control) was obtained from AbCam (Cambridge, MA, USA). Stock antibody was aliquoted and maintained at $-70^{\circ} \mathrm{C}$. Stock antibody was used at a final concentration equal to AdipoR1 antibodies $(1 \mu \mathrm{g} / \mu \mathrm{L})$ as a nonspecific, IgG antibody control for immunoaffinity capture experiments.

\section{Immunomass Spectrometric Analysis}

Polyclonal and monoclonal antibodies against epitopes of the AdipoR1-CTF and the antibody control were immobilized on pre-activated PS20 chip surfaces (Bio-Rad Laboratories). Plasma samples from healthy and diabetic patients as well as peptide controls were randomized in duplicate across anti-AdipoR1/PS20 chips. The peptide standard and cross-reactive protein/peptide species from plasma samples were captured on the chip and their molecular weights were determined by immunomass spectrometry.

\section{Statistics}

The selectively captured protein peaks were normalized. Using the Biomarker Wizard Data Analysis Tool, an unsupervised cluster analysis was performed. Signal/noise ratio cutoffs of 10 (for polyclonal experiments) and 5 (for monoclonal experiments) were used to select peaks with the 
greatest difference between healthy and diabetic patient groups. Peak intensities were compared among the groups, and $p$ values were calculated using Biomarker Wizard. Differences in peak intensities between the study groups with $p<0.05$ were considered significant.

\section{Results}

Initial Discovery Using Polyclonal Anti-AdipoR1

Initial immunoaffinity capture studies using the polyclonal antibody specific for the $19 \mathrm{C}$-terminal amino acids of AdipoR1 revealed a number of captured peptides and proteins (Fig. 1). From the peptide standard and the plasma samples collected from healthy $(n=5)$ and diabetic patients $(n=5)$, AdipoR1 and AdipoR1-ligand complexes from 2 to $\sim 33 \mathrm{kD}$ were captured on the polyclonal AdipoR1/PS20 chips. Profile analysis from AdipoR1/PS20 chips revealed 15 cross-reactive peaks, five of which were of significant interest at $\mathrm{m} / \mathrm{z}$ of $3,902,7,812,14,636,28,483$, and 28,688, respectively (Fig. 1). Cluster analysis suggested the 3,902 and 7,812 proteins were significantly increased in healthy patients $(p<0.0001)$, while the other three (14 and $28 \mathrm{kD})$ proteins were increased in diabetics $(p<0.01$; Table 1). Although not considered to be significantly different between the control and diabetic groups, the other ten peaks identified may represent different receptor-ligand forms captured by the AdipoR1/PS20 chip. Subsequent immunoaffinity capture analysis using the monoclonal AdipoR1 antibody and more patient samples helped to characterize the identity and significance of the captured, cross-reactive species to AdipoR1.

Confirmation of Discovered Fragment by Monoclonal Anti-AdipoR1 Antibody

As indicated above, the polyclonal antibody captured several peaks, including the 3,902-Da fragment. As illustrated in Fig. 3, this fragment is consistent with the last 35 amino
Table 1 Immunoaffinity capture of cross-reactive peaks with polyclonal AdipoR1

\begin{tabular}{|c|c|c|c|c|}
\hline \multirow[t]{2}{*}{$m / z$} & \multicolumn{2}{|c|}{ Average signal to noise } & \multirow{2}{*}{$\begin{array}{l}\text { Fold } \\
\text { difference }\end{array}$} & \multirow{2}{*}{$\begin{array}{l}\text { Significance } \\
\text { ( } p \text { value) }\end{array}$} \\
\hline & $\begin{array}{l}\text { Healthy } \\
(n=5)\end{array}$ & $\begin{array}{l}\text { Diabetic } \\
(n=5)\end{array}$ & & \\
\hline 3,902 & 10.11 & 2.10 & 4.8 & 0.00067 \\
\hline 7,812 & 5.11 & 1.21 & 4.2 & 0.00016 \\
\hline 14,636 & 1.93 & 3.71 & 1.9 & 0.01261 \\
\hline 28,483 & 5.37 & 7.99 & 1.5 & 0.04125 \\
\hline 28,688 & & & & 0.03429 \\
\hline
\end{tabular}

$\mathrm{m} / \mathrm{z}$ mass/charge ratio

acids of AdipoR1 containing the transmembrane domain with a calculated molecular weight of 3,824 Da. Therefore, to assure that the captured CTF was in fact the extracellular fragment of AdipoR1, a monoclonal antibody targeting only the last 25 C-terminal amino acids (clone 444-1D12.1H7) was generated and used for immunoaffinity capture studies to confirm detection of these fragments in plasma from healthy $(n=10)$ and diabetic $(n=10)$ subjects.

Using this monoclonal antibody to the 25 mer, two specific C-terminal AdipoR1 fragments, 3,381 and 3,498 Da, were captured on the monoclonal AdipoR1/PS20 chips. Inherent to the clean capture of fragments with monoclonal antibody, the other immunoreactive peaks captured using polyclonal antibody were less evident. Both fragments captured demonstrated significant differences between healthy and diabetic subjects $3,381 \mathrm{Da}(p<0.01)$ and $3,498 \mathrm{Da}(p<0.05$; Fig. 2). Notably, as observed in the preliminary polyclonal capture experiment, both fragments were consistently captured from plasma samples of healthy patients. The captured corresponding masses surpass the total mass of the 25 amino acid, extracellular CTF, and approximate an AdipoR1 fragment 30-31 amino acids in length (Fig. 3) with a calculated molecular weight of 3,475 Da for the 32-mer peptide. From this point, we defined this particular peptide detected in plasma as AdipoR1 CTF.

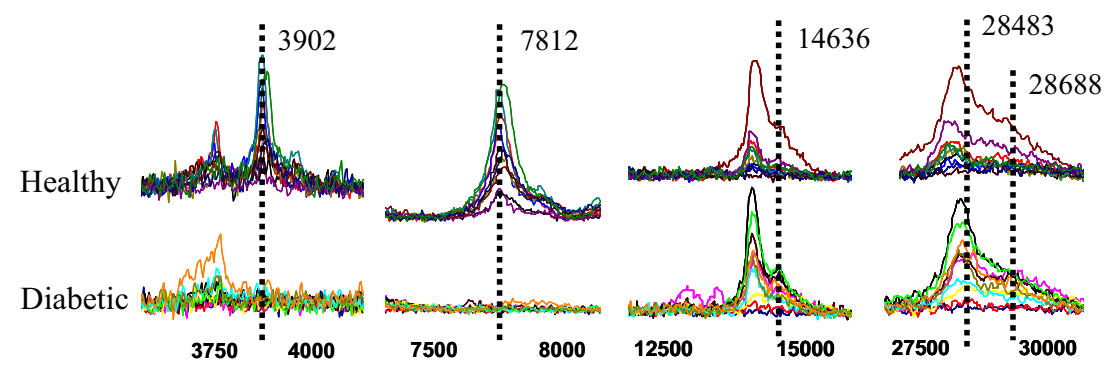

Fig. 1 Polyclonal AdipoR1-related immunoreactivity in healthy and diabetic plasma. Polyclonal captured low molecular weight peaks $(3,902 ; 7,812)$ are present in healthy subjects, but absent in diabetics $(n=5)$. By contrast, polyclonal captured high molecular weight peaks $(14,636 ; 28,483 ; 28,688)$ are present in diabetics, but less abundant in healthy subjects $(n=5)$ 


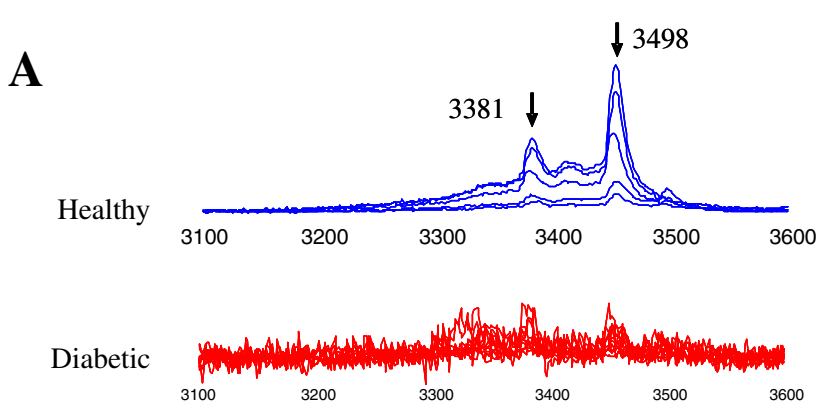

B

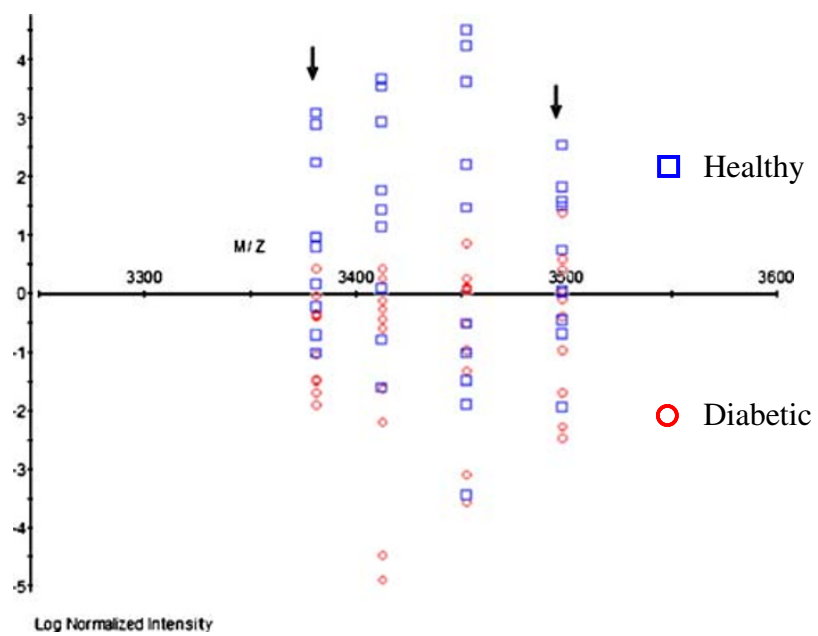

Fig. 2 Monoclonal AdipoR1-related immunoreactivity in healthy and diabetic plasma. Low molecular weight scan revealed significant differences between healthy subjects and diabetics. Representative scans (a) of five of ten from each group are shown. Note: Scan noise is greater in diabetic scans shown in a due to a fivefold increase in the peak intensity scale. b Cluster analysis of monoclonal captured fragments from the whole group of healthy $(n=10)$ and diabetic $(n=10)$ patients indicate that 3,381 and 3,498 peaks are significantly associated with healthy individuals and absent or less abundant in diabetics

\section{Discussion}

It is well recognized that diabetes mellitus is a group of metabolic diseases characterized by hyperglycemia resulting from defects in insulin production, secretion, action, or combination thereof [20-22]. The American Diabetes Association has systematically developed clinical practice guidelines for the diagnosis and classification of diabetes. Current recommendations for clinical laboratory testing include fasting plasma glucose, glucose intolerance test, and the oral glucose tolerance test. As evidenced by this current clinical practice, the main criterion for making a diagnosis of diabetes is an elevated glucose level. However, recent evidence suggests that elevated blood glucose may not be as strongly associated with diabetes as once believed to be. A clinical study by Nichols and colleagues [23] evaluated the risk of diabetes associated with normal fasting glucose levels (less than $100 \mathrm{mg} / \mathrm{dL}$ ) in over 46,578 patients. After nearly 7 years of follow-up, these subjects developed type 2 diabetes at a rate of $1 \%$ per year. After controlling for other risk factors, each milligrams per deciliter rise in fasting blood glucose corresponded to an increased diabetes risk by $6 \%$, and subjects with glucose levels of 95-99 mg/dL were 2.33 times more likely to develop diabetes compared with those subjects with fasting blood glucose levels of less than $85 \mathrm{mg} / \mathrm{dL}$. Despite the usefulness of clinical practice guidelines, these clinical study findings would appear to stress a need to redefine the criteria for diagnostic confirmation of diabetes and to seek other candidate biomarkers that may be more useful in predicting diabetes development.

Adiponectin and the interplay between its associated receptor system (AdipoR1 and AdipoR2) have been recognized as potential mechanisms for obesity, insulin resistance, type 2 diabetes, and the metabolic syndrome. This hormone-receptor system has been shown to play an important role in energy homeostasis via its direct insulinsensitizing actions on tissues especially liver and skeletal muscle [13-16]. In order to understand the role of adiponectin and its receptor fragments in disease, we characterized their cross-reactivities in plasma from healthy and diabetic patients using immunomass spectrometry.

From a peptide standard as well as healthy and diseased plasma samples, AdipoR1 fragments with molecular weights of $\sim 3.5 \mathrm{kD}$ were captured on the chip by polyclonal and monoclonal AdipoR1 antibodies. Differential binding patterns between healthy and diabetic plasma were identified, suggesting inherent differences in AdipoR1 fragments and/or AdipoR1-adiponectin (receptor-ligand) complexes between these two groups. Ultimately, through this study, we were able to identify a specific 31-32 amino acid C-terminal AdipoR1 fragment in healthy patients that was absent in diabetic individuals using a monoclonal antibody targeted to capture the 25-mer Adipo-R1 CTF. Altogether, these data confirmed that AdipoR1-related fragments can be found in healthy control and diabetic patients.

The two low molecular weight fragments $(3,902$ and 7,812) identified by the polyclonal AdipoR1 study likely represent the CTF of AdipoR1, which may also include part of the transmembrane domain. Additionally, given that the extracellular, C-terminal domain contains a cysteine residue, once this portion of R1 is shed or turned over, two AdipoR1 C-terminal fragments could potentially dimerize. This was also an observed phenomenon with the synthesized C-terminal control AdipoR1 peptide used in this study. Thus, the 7,812 fragment could potentially represent the homodimerization of two C-terminal domains in healthy individuals during normal receptor turnover. Alternatively, the other higher molecular weight masses observed $(14,636$, 28,483, and 28,688) were identified and significantly 
Fig. 3 AdipoR1 receptor. The AdipoR1 receptor is an atypical seven-transmembrane domain, $\mathrm{G}$ protein-coupled receptor. As shown, the AdipoR1 receptor is unique in that the $\mathrm{C}$-terminal end is located on the extracellular side of the membrane and $\mathrm{N}$-terminal on the intracellular side. AdipoR1 C-terminal fragments of interest are highlighted including the 19-, 25-, 32-, and 35-amino acid fragments: 19-aa fragment represents target for polyclonal antibody, 25-aa fragment represents the target for the monoclonal antibody, 32-aa fragment represents the clinical fragment captured by the monoclonal AdipoR1 immunoaffinity, and 35-aa fragment represents the polyclonal AdipoR1 immunoaffinity captured fragment

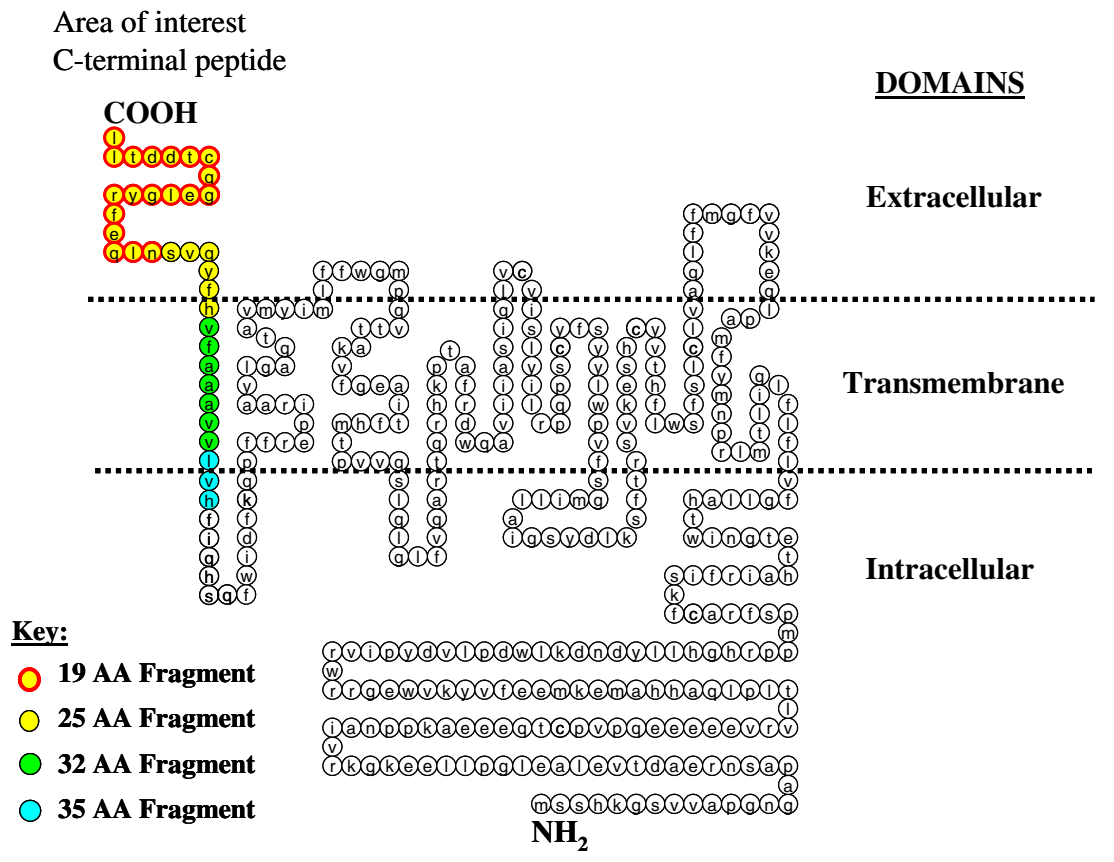

associated with the presence of a diabetic state. Further testing revealed that the later three masses corresponded to adiponectin in the globular (gAdpn) and full-length (fAdpn) forms binding as cross-reactive species to the polyclonal. To help rule out CTF-mediated immunoaffinity capture of adiponectin by the polyclonal antibody in diabetics, further capture work was performed after co-incubation of adiponectin (gAdpn and fAdpn forms) and purified CTF peptide. Notably, these coincubation experiments performed indicated that a synthesized AdipoR1 fragment did not bind to adiponectin in either form and the repeat immunoaffinity capture using a polyclonal antibody for adiponectin confirmed these findings (data not shown).

The subsequent monoclonal work identified a specific 31-32 amino acid (3,381 and 3,498 Da) AdipoR1 CTF in healthy patients that was absent in diabetic individuals. A potential explanation of differences in fragmentation patterns between these two study groups is the lack of multimer adiponectin during the insulin resistant diabetic state and a high rate of receptor turnover in insulinresponsive, healthy individuals.

As demonstrated, immunoaffinity capture followed by mass spectrometric determination of the molecular weights of the captured peptides and proteins can be a powerful biomarker discovery tool. In this study, since the molecular weights of a number of the discovered species were less than $10 \mathrm{kD}$, Western blot analysis would not have resolved the discovered peaks. Likewise, due to the many different cross-reactive forms being present in each sample, using ELISA, the observed differences in AdipoR1 crossreactivity between healthy and diabetic plasma may have been masked. Therefore, we conclude that immunoaffinity mass spectrometry is a powerful and useful tool to determine antibody specificity and, more specifically, the molecular weight and fundamental identity of ligands or cross-reactive ligands. Further research and biomarker discovery studies using proteomic tools such as immunoaffinity capture are necessary to continue to help define and understand the potential role of adipokines and AdipoR1related fragments in healthy and disease states.

Potentially cell surface receptor fragments released in vivo can serve as novel sources of biomarkers indicative of receptor regulation in disease conditions. In fact, there have been several published studies in which fragments of transmembrane proteins have been generated via ectodomain cleavage. For instance, a $100-\mathrm{kD}$ fragment of the N-terminal ectodomain of amyloid precursor protein is known to be generated by $\alpha$-secretase, which cleaves the protein at a site that precludes the formation of the amyloid plaque-forming $\beta \mathrm{A} 4$ peptide [24, 25]. As reviewed by Fortini [26], binding of ligands of the Delta/Serrate/Jagged family to the Notch receptor triggers the cleavage of the extracellular domain of Notch by tumor necrosis factor- $\alpha$-converting enzyme/a disintegrin and metalloprotease-17 (TACE/ADAM17). The N-terminal ectodomain of the ErbB-4 receptor can also be cleaved by TACE/ADAM17 to yield an m80 fragment, which has been found to be associated with the full-length ErbB-2 receptor [27]. More recently, a study by Szklarczyk and colleagues [28] demonstrated that the zinc-dependent matrix metalloproteinase-7 can cleave the NR1 subunit of the $N$-methyl-D-aspartate (NMDA) receptor at a site within the extracellular ligand binding domain, leading to impairment of NMDA-stimulated calcium flux in cortical neurons and possible alteration of neural transmission. In addition, 
stable N-terminal fragments of the NR2 subunit of the NMDA receptor have been generated via proteolytic cleavage $[29,30]$, and autoantibodies against these fragments have been detected in the blood of transient ischemic attack and stroke patients [31]. The detection of these autoantibodies in human serum indicates that these $\mathrm{N}$-terminal fragments can cross the blood-brain barrier and enter the bloodstream [31].

Furthermore, investigating cell responses and differences in signaling pathways have led to a better understanding of the role of the adiponectin-receptor axis in disease. Stimulation of adiponectin receptors enhances glucose uptake and fatty acid oxidation through activation of 5'adenosine monophosphate-activated protein kinase and parallel phosphorylation of acetyl coenzyme A carboxylase [15]. Binding of adiponectin to its receptors has been shown to activate peroxisome proliferator-activated receptor $\alpha$ and subsequent stimulation of fatty acid oxidation and reduction of triglyceride content in muscle and liver [12, 14]. The adiponectin receptors respond differently to the various forms of adiponectin, which are primarily either full length or globular (i.e., fAdpn or gAdpn) [32]. Adiponectin has been shown to regulate inflammation and protect against atherogenesis by antagonizing the pro-inflammatory effects of tumor necrosis factor- $\alpha[14,33-37]$. Due to C1q-like, collagenous domains, fAdpn monomers can aggregate into multimers of low, medium, and high (HMW) molecular weight forms, with the HMW form accounting for half of total adiponectin in normal individuals [32, 38-40]. A proteolytic cleavage product of adiponectin is known as gAdpn. AdipoR2 only reacts to the fAdpn form while AdipoR1 is more responsive to the gAdpn form [40].

As reviewed by Tilg and Moschen [11], expansion of adipose tissue during weight gain can lead to release of proinflammatory cytokines and recruitment of macrophages, resulting in a low-grade inflammatory state. An increase of these pro-inflammatory mediators is also accompanied with a decline in the release of adiponectin from adipose tissue. In conjunction with the observed decrease in adiponectin levels, obesity may either directly lead to decreased expression of AdipoR, or it may indirectly reduce expression of these receptors via producing hyperinsulinemia [41]. A study of fasted and re-fed $o b / o b$ mice [17] revealed that expression of AdipoR1 and Adipo R2 in skeletal muscle and liver, respectively, were increased during fasting, but were decreased to their original fed state levels during refeeding. Furthermore, when hypoinsulinemia/ hyperglycemia was induced by streptozotocin, AdipoR1 and R2 mRNA expression were increased in these mice, and notably, after treatment with insulin, expression levels of both receptors were reduced to nearly the original values. Under conditions of hyperinsulinemia and hyperglycemia, an in vitro study [42] of rat skeletal muscle cells demonstrated a $50 \%$ decrease in AdipoR1 mRNA levels and R1 expression, which correlated with reduced metabolic effects of gAdpn in these skeletal muscle cells. In addition, hyperinsulinemia alone induced a threefold increase in AdipoR2 expression and enhanced the metabolic effects of fAdpn in these cells. This alteration in AdipoR expression and the variation in response to the gAdpn and fAdpn forms are the basis for the proposed adiponectin resistance in type 2 diabetes, and this type of resistance can lead to the creation of a vicious cycle that exacerbates the development of diabetes [41].

In our preliminary experiments using Western blotting and an ELISA polyclonal antibody method, we detected fragments of the extracellular $\mathrm{C}$ terminus of AdipoR1 in human plasma and urine [19]. However, due to limitations associated with the use of a polyclonal antibody, the specific fragments could not be distinguished. To confirm the identity and characterize the potential AdipoR1's specific fragments in plasma, we used a novel approach by combining mass spectrometry and monoclonal antibody cross-reactivity for rapid characterization of plasma proteins in pursuit of discovery of new biomarkers [43]. This technique allows for a highly sensitive approach to study antibody-ligand interactions, which we have previously utilized to study the interaction of various forms of troponin I to its antibodies [44]. Having the monoclonal antibody against AdipoR1 CTF will allow the development of specific immunoassays to be used in assessing the utility of this putative biomarker for diabetes.

Acknowledgement Supported in part by the NIEHS award number is P30ES014443 titled: Center for Environmental Genomics and Integrative Biology to University of Louisville School of Medicine.

\section{References}

1. Arita Y, Kihara S, Ouchi N, et al. Paradoxical decrease of an adipose-specific protein, adiponectin, in obesity. Biochem Biophys Res Commun. 1999;257(1):79-83.

2. Daimon M, Oizumi T, Saitoh T, et al. Decreased serum levels of adiponectin are a risk factor for the progression to type 2 diabetes in the Japanese population: the Funagata study. Diabetes Care. 2003;26(7):2015-20.

3. Duncan BB, Schmidt MI, Pankow JS, et al. Adiponectin and the development of type 2 diabetes: the atherosclerosis risk in communities study. Diabetes. 2004;53(9):2473-8.

4. Krakoff J, Funahashi T, Stehouwer CDA, et al. Inflammatory markers, adiponectin and risk of type 2 diabetes in the Pima Indian. Diabetes Care. 2003;26(6):1745-51.

5. Lindsay RS, Funahashi T, Hanson RL, et al. Adiponectin and development of type 2 diabetes in the Pima Indian population. Lancet. 2002;360:57-8.

6. Ryo M, Nakamura T, Kihara S, et al. Adiponectin as a biomarker of the metabolic syndrome. Circulation Journal. 2004;68(11):97581. 
7. Snehalatha C, Mukesh B, Simon M, Viswanathan V, Haffner SM, Ramachandran A. Plasma adiponectin is an independent predictor of type 2 diabetes in Asian Indians. Diabetes Care. 2003;26 (12):3226-9.

8. Spranger J, Kroke A, Mohlig M, et al. Adiponectin and protection against type 2 diabetes mellitus. Lancet. 2003;361:226-8.

9. Yamamoto Y, Hirose H, Saito I, Nishikai K, Saruta T. Adiponectin, an adipocyte-derived protein, predicts future insulin resistance: twoyear follow-up study in Japanese population. J Clin Endocrinol Metab. 2004;89(1):87-90.

10. Yatagai T, Nagasaka S, Taniguchi A, et al. Hypoadiponectinemia is associated with visceral fat accumulation and insulin resistance in Japanese men with type 2 diabetes mellitus. Metabolism. 2003;52(10):1274-8.

11. Tilg H, Moschen AR. Adipocytokines: mediators linking adipose tissue, inflammation and immunity. Nat Rev Immunol. 2006;6:77283.

12. Yamauchi $\mathrm{T}$, Kamon $\mathrm{J}$, Ito $\mathrm{Y}$, et al. Cloning of adiponectin receptors that mediate antidiabetic metabolic effects. Nature. 2003;423:762-9.

13. Kadowaki T, Yamauchi T, Kubota N, Hara K, Ueki K, Tobe K. Adiponectin and adiponectin receptors in insulin resistance, diabetes and the metabolic syndrome. J Clin Invest. 2006;116 (7): 1784-92.

14. Yamauchi T, Hara K, Kubota N, et al. Dual Roles of adiponectin/ acrp30 in vivo as an anti-diabetic and anti-atherogenic adipokine. Curr Drug Targets Immune Endocr Metabol Disord. 2003;3 (4):243-53.

15. Yamauchi T, Kamon J, Minokoshi Y, et al. Adiponectin stimulates glucose utilization and fatty-acid oxidation by activating AMP-activated protein kinase. Nature Medicine. 2002;8(11):128895.

16. Yamauchi T, Kamon J, Waki $\mathrm{H}$, et al. The fat-derived hormone adiponectin reverses insulin resistance associated with both lipoatrophy and obesity. Nature Medicine. 2001;7(8):941-6.

17. Tsuchida A, Yamauchi $\mathrm{T}$, Ito $\mathrm{Y}$, et al. Insulin/Foxol pathway regulates expression levels of adiponectin receptors and adiponectin sensitivity. J Biol Chem. 2004;279(29):30817-22.

18. Yamauchi T, Nio Y, Maki T, et al. Targeted disruption of AdipoR1 and AdipoR2 causes abrogation of adiponectin binding and metabolic actions. Nat Med. 2007;13(3):332-9.

19. Pugia MJ. Inventor detection of soluble adiponectin receptor peptides and use in diagnostics and therapeutics. United States patent PCT/US2006061555. 2005 Dec 4.

20. American Diabetes Association. Diagnosis and classification of diabetes mellitus. Diabetes Care. 2007;30(Supplement 1):S42-7.

21. American Diabetes Association. Standards of medical care in diabetes - 2007. Diabetes Care. 2007;30(Supplement 1):S4-41.

22. The Expert Committee on the Diagnosis and Classification of Diabetes Mellitus. Follow-up report on the diagnosis of diabetes mellitus. Diabetes Care. 2003;26(11):3160-7.

23. Nichols GA, Hillier TA, Brown JB. Normal fasting plasma glucose and risk of type 2 diabetes diagnosis. Am J Med. 2008;121(6):51924.

24. Esch FS, Keim PS, Beattie EC, et al. Cleavage of amyloid beta peptide during constitutive processing of its precursor. Science. 1990;248(4959):1122-4.

25. Hooper NM, Karran EH, Turner AJ. Membrane protein secretases. Biochem J. 1997;321(Pt 2):265-79.

26. Fortini ME. Gamma-secretase-mediated proteolysis in cellsurface-receptor signalling. Nat Rev Mol Cell Biol. 2002;3(9):67384.
27. Cheng QC, Tikhomirov O, Zhou W, Carpenter G. Ectodomain cleavage of ErbB-4: characterization of the cleavage site and $\mathrm{m} 80$ fragment. J Biol Chem. 2003;278(40):38421-7.

28. Szklarczyk A, Ewaleifoh O, Beique JC, et al. MMP-7 cleaves the NR1 NMDA receptor subunit and modifies NMDA receptor function. FASEB J. 2008;22(11):3757-67.

29. Simpkins KL, Guttmann RP, Dong Y, et al. Selective activation induced cleavage of the NR2B subunit by calpain. J Neurosci. 2003;23(36):11322-31.

30. Yuan $\mathrm{H}$, Vance KM, Junge CE, et al. The serine protease plasmin cleaves the amino-terminal domain of the NR2A subunit to relieve zinc inhibition of the N-methyl-D-aspartate receptors. J Biol Chem. 2009;284(19):12862-73.

31. Dambinova SA, Khounteev GA, Izykenova GA, Zavolokov IG, Ilyukhina AY, Skoromets AA. Blood test detecting autoantibodies to N-Methyl-D-aspartate neuroreceptors for evaluation of patients with transient ischemic attack and stroke. Clin Chem. 2003;49 (10): $1752-62$.

32. Ebinuma H, Miyazaki O, Yago $H$, Hara $K$, Yamauchi $T$, Kadowaki T. A novel ELISA system for selective measurement of human adiponectin multimers by using proteases. Clin Chim Acta. 2006;372(1-2):47-53

33. Matsuura F, Oku H, Koseki M, et al. Adiponectin accelerates reverse cholesterol transport by increasing high density lipoprotein assembly in the liver. Biochem Biophys Res Commun. 2007;358 (4):1091-5.

34. Ouchi N, Kihara S, Arita Y, et al. Novel modulator for endothelial adhesion molecules: adipocyte-derived plasma protein adiponectin. Circulation. 1999;100(25):2473-6.

35. Ouchi N, Kihara S, Arita Y, et al. Adipocyte-derived plasma protein, adiponectin, suppresses lipid accumulation and class a scavenger receptor expression in human monocyte-derived macrophages. Circulation. 2001;103(8):1057-63.

36. Ouchi N, Kihara S, Arita Y, et al. Adiponectin, an adipocyte-derived plasma protein, inhibits endothelial NF-kB signaling through a cAMP-dependent pathway. Circulation. 2000;102(11):1296-301.

37. Whitehead JP, Richards AA, Hickman IJ, Macdonald GA, Prins JB. Adiponectin - a key adipokine in the metabolic syndrome. Diabetes Obes Metab. 2006;8:264-80.

38. Pajvani UB, Du X, Combs TP, et al. Structure-function studies of the adipocyte-secreted hormone Acrp30/adiponectin: implications for metabolic regulation and bioactivity. J Biol Chem. 2003;278 (11):9073-85.

39. Scherer PE, Williams S, Fogliano M, Baldini G, Lodish HF. A novel serum protein similar to $\mathrm{C} 1 \mathrm{q}$, produced exclusively in adipocytes. J Biol Chem. 1995;270(45):26746-9.

40. Waki H, Yamauchi T, Kamon J, et al. Impaired multimerization of human adiponectin mutants associated with diabetes: molecular structure and multimer formation of adiponectin. J Biol Chem. 2003;278(41):40352-63.

41. Kadowaki T, Yamauchi T. Adiponectin and adiponectin receptors. Endocr Rev. 2005;26(3):439-51.

42. Fang $X$, Palanivel $R$, Zhou $X$, et al. Hyperglycemia- and hyperinsulinemia-induced alteration of adiponectin receptor expression and adiponectin effects in L6 myoblasts. J Mol Endocrinol. 2005;35(3):465-76.

43. Zhu Y, Valdes R Jr, Jortani SA. Application of bioaffinity mass spectrometry for analysis of ligands. Ther Drug Monit. 2005;27 (6):694-9.

44. Franke DD, Valdes R Jr, Jortani SA. A method for profiling crossreactivities of cardiac troponin I antibodies used in current diagnostic assays. Clin Chem. 2007;53(6):A19. 